\title{
Study on Surface Evenness of Super-high-thickness Cutting in High-speed Wire Electrical Discharge Machining
}

\section{Cong Deng}

Nanjing university of Aeronautics and Astronautics

Zhidong Liu ( $\square$ liutim@nuaa.edu.cn )

Nanjing University of Aeronautics and Astronautics

Ming Zhang

Nanjing university of Aeronautics and Astronautics

Hongwei Pan

Nanjing university of Aeronautics and Astronautics

Mingbo Qiu

Nanjing university of Aeronautics and Astronautics

\section{Research Article}

Keywords: WEDM, super-high thickness, surface evenness, working fluid, 2000 mm workpiece

Posted Date: March 18th, 2021

DOI: https://doi.org/10.21203/rs.3.rs-312872/v1

License: (c) (i) This work is licensed under a Creative Commons Attribution 4.0 International License. Read Full License 


\title{
Study on surface evenness of super-high-thickness cutting in high-speed wire electrical discharge machining
}

\author{
Cong Deng ${ }^{1}$, Zhidong Liu ${ }^{1}$, Ming Zhang ${ }^{1}$, Hongwei Pan ${ }^{1}$, Mingbo Qiu ${ }^{1}$ \\ (College of Mechanical and Electrical Engineering, \\ Nanjing university of Aeronautics and Astronautics, Nanjing, 210016)
}

\begin{abstract}
Surface machined by high-speed wire electrical discharge machining (HS-WEDM) at super-high thickness (more than $1000 \mathrm{~mm}$ ) cutting suffers from uneven surface, a major problem that has been investigated in this paper. According to the analysis, as wire frame span increases, the rigidity of the wire electrode decreases, and under the action of discharge explosive force, wire electrode vibration intensifies. As a result, the machining stability inevitably decreases. However, the core problem is whether there is enough working fluid in the slit to dampen and absorb the vibration of the wire electrode so as to ensure the positional stability of the wire electrode. To verify the above point of view: first, the wire guide and gravity take-up with bidirectional tension in the wire feeding system were installed to improve the positional accuracy of the wire electrode; second, to improve the flow of the working fluid into the slit, the slit width was increased by improving the working fluid and a medium carrier with a higher melting point and vaporization point can reduce the vaporization of the working fluid in the slit as much as possible. The experiment showed that the outlet flow of the improved working fluid is $56.72 \%$ higher than that of the original working fluid when cutting a $750 \mathrm{~mm}$ thick workpiece, which increases the damping and vibration absorption effect of the working fluid on the wire electrode in the long and narrow gap. After the above measures were implemented, super-high thickness cutting can be carried out continuously and steadily, the surface evenness was significantly improved, and the workpiece with a thickness of $2000 \mathrm{~mm}$ was cut successfully.
\end{abstract}

Keywords WEDM, super-high thickness, surface evenness, working fluid, $2000 \mathrm{~mm}$ workpiece

\section{Introduction}

Super-high-thickness machining has broad application prospects in nuclear energy, aerospace and other fields ${ }^{[1]}$. since the wire electrode is travelling at a high speed in high-speed wire electrical discharge machining (HS-WEDM), the working fluid can be attached to the wire electrode and the working fluid can be easily brought into the processing area, which can ensure inter-electrode cooling, chip removal, and deionization, and provide cutting capability of superhigh thickness workpieces (more than $1000 \mathrm{~mm}$ ) ${ }^{[2]}$. In the $1980 \mathrm{~s}$, Li et al. ${ }^{[3]}$ began to explore the theory and technology of high thickness cutting in HS-WEDM and machined a workpiece with a thickness of $805 \mathrm{~mm}$. In 2007, Wan ${ }^{[4]}$ designed a new wire electrode, wire tension device, and wire guide to allow for cutting at thicknesses up to $1000 \mathrm{~mm}$. In 2017, Li et al. [5] conducted theoretical analysis and experimental verification on the inter-electrode discharge energy and working medium flow in super-high thickness cutting, realized some important conditions for super-high thickness, and achieved cutting of a $1500 \mathrm{~mm}$ thick workpiece. In recent years, with further research and application of intelligent high-frequency pulse power supply and compound working fluid for super-high thickness cutting, it is not difficult for HS-WEDM to realize ultra-high thickness workpiece cutting. However, ensuring surface evenness of super-high thickness workpiece cutting is still a significant challenge. Improved surface evenness for super-high thickness cutting workpieces is an important developmental direction to expand the application scope of HS-WEDM machine tools ${ }^{[1]}$. At present, the uneven stripes appear on the workpiece surface after super-high thickness processing, which seriously damages the surface quality of the workpiece, and greatly limits the applicability of

*Corresponding author.

E-mail address: liutim@nuaa.edu.cn (Zhidong Liu) 
the HS-WEDM machine tool with high surface requirements in super-high thickness cutting. The main purpose of this paper is to study the influence of different working fluids on the surface evenness by changing the working fluid of super-high thickness cutting, so as to improve the surface quality of superhigh thickness cutting workpieces, and further expand the application space of super-high thickness cutting in other applications.

\section{Experimental system}

The experiment was conducted using a HF500A HS-WEDM machine tool, where the maximum cutting thickness can reach $2000 \mathrm{~mm}$, as shown in Fig. 1. On the basis of the original gravity take-up on the upper frame, another gravity take-up was installed on the lower frame, so gravity take-up with bidirectional tension is formed. In addition, two wire guides were installed in the machining area. The workpiece with a thickness of 1000 mm was machined according to the experimental conditions and parameters of Table 1, and its surface evenness was observed and measured after cutting.

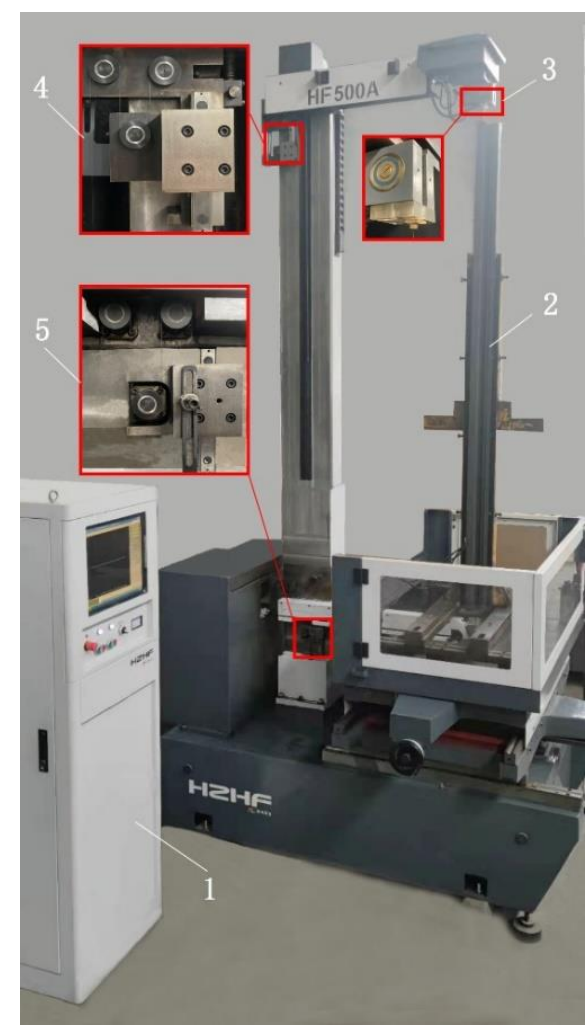

1 Control cabinet 2 Workpiece 3 Wire guide

4 Upper gravity take-up 5 Lower gravity take-up Fig.1 Photograph of the experimental equipment
Table 1 Experimental parameters

\begin{tabular}{|c|c|}
\hline Item & parameter \\
\hline Material & 45\#, Thickness:1000mm \\
\hline wire & $\begin{array}{l}\text { Molybdenum wire, diameter }= \\
0.18 \mathrm{~mm} \text {, length }=300 \mathrm{~m}\end{array}$ \\
\hline Wire tension & 10N (upper gravity take-up) \\
\hline Working fluid & JR1A, 1:20 \\
\hline Cutting parameter & $\begin{array}{l}\text { Pulsewidth, } 80 \mu s ; \text { Duty cycle, } 1: 8 \text {; } \\
\text { MOS tube number, } 12\end{array}$ \\
\hline Wire speed & $12 \mathrm{~m} / \mathrm{s}$ \\
\hline Average current & $4.5 \mathrm{~A}$ \\
\hline
\end{tabular}

Figure 2 shows the cutting surface of the workpiece with a thickness of $1000 \mathrm{~mm}$. Deep and dense streaks are often formed on the upper and lower ends of the workpiece. If the machining parameters are not adjusted properly, the streaks will run through the entire cutting surface. The depth is up to submillimeter level, which seriously damages the surface quality of the workpiece.

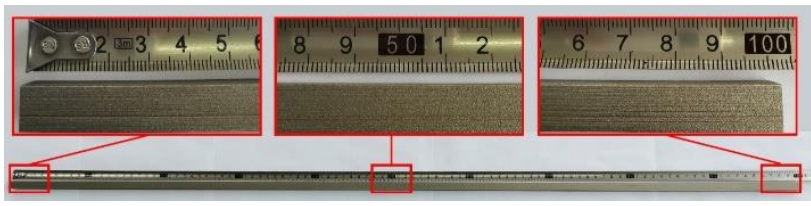

Fig.2 The overall and partially magnified photograph of the $1000 \mathrm{~mm}$ thick workpiece

\section{Flow model of the inter-electrode working fluid}

To analyze the cause of streak formation, a flow model of inter-electrode working fluid was established to analyze the relationship between streaks formation and machining parameters. In HS-WEDM, the shear flow of the working fluid in inter-electrode flow field is three orders of magnitude larger than that of the pressure difference flow ${ }^{[4]}$, so this paper primarily analyzes the shear flow of the working fluid. Suppose that the radius of the wire electrode is $r_{1}$, its axial moving velocity is $v_{0}$, and the slit width $\delta=2 \mathrm{r}_{2}$, then the shear flow velocity distribution $U$ of the working fluid is ${ }^{[4]}$ :

$$
\mathrm{U}=\frac{v_{0}}{\operatorname{In}\left(r_{1} / r_{2}\right)} \operatorname{In} \frac{r}{r_{2}}
$$

It is assumed that the working medium flows in a concentric cylindrical annular gap centered on the axis of the wire electrode, as shown in Fig. 3. A finite element ring with radius $\mathrm{r}$ and width $\mathrm{dr}$ is selected on the slit section, and its area is $d s=2 \pi r d r$. By integrating 
the shear flow velocity of the working fluid, the flow $\mathrm{Q}$ of the working fluid can be obtained as:

$$
\begin{aligned}
Q & =\int U d s=2 \pi \int_{r_{1}}^{r_{2}} \frac{v_{0}}{\operatorname{In}\left(r_{1} / r_{2}\right)} \operatorname{In} \frac{r}{r_{2}} r d r \\
& =\pi v_{0}\left(\frac{r_{1}^{2}-r_{2}^{2}}{2 \operatorname{In}\left(r_{1} / r_{2}\right)}-r_{1}^{2}\right)
\end{aligned}
$$

It can be seen from equation (2) that the flow $\mathrm{Q}$ of the inter-electrode working fluid increases with increasing wire travelling speed $\mathrm{v}_{0}$ and slit width $\delta$.

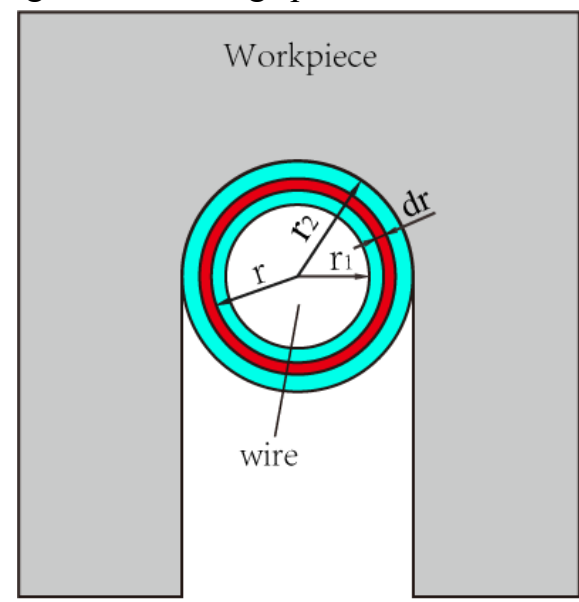

Fig. 3 Flow model of the working fluid in the annular gap of a concentric cylinder

\section{Causes of uneven surfaces for super-high thickness machining}

For super-high thickness WEDM, the rigidity of the wire electrode decreases sharply due to the increasing length of the wire electrode in the processing area, so the vibration of the wire electrode greatly increases when it is slightly disturbed, and the working fluid is difficult to enter into the depth of the cutting slit, which leads to the greater difference between the interelectrode discharge state and the conventional thickness cutting. Therefore, the machining stability of super-high thickness machining is far lower than that of conventional thickness cutting.

\subsection{Effect of the inter-electrode working fluid}

In the process of super-high thickness machining, the working fluid not only plays the role of interelectrode cooling, washing and deionization, but can also absorb and damp the vibration of the wire electrode [6]. In super-high thickness machining, due to the increased cutting path, the working fluid brought into the cutting slit by the high-speed movement of the wire electrode will continue to vaporize under the action of high temperature generated by discharge, and the slit outlet will have inadequate working fluid due to the lack of timely supplementation. When the wire electrode travels downward, the lower end of the slit will lack working fluid, and when the wire electrode travels upward, the upper end of the workpiece will lack the working fluid. Therefore, the upper and lower ends of the cutting slit will always be in a bad discharge state due to the lack of working fluid. The abnormal discharge environment and lack of damping of the liquid medium will cause the vibration of the wire electrode to sharply increase, which is also consistent with the phenomenon that dense streaks will appear at both ends of the workpiece.

To verify the deterioration of the discharge environment at the lower end of the slit when the wire electrode is travelling downward, the following experimental scheme was designed. The workpiece was divided into workpiece 1 and workpiece 2 in the experiment. The thickness of workpiece 1 was $750 \mathrm{~mm}$ and that of workpiece 2 was $50 \mathrm{~mm}$. Workpieces 1 and 2 are separated by an insulator with a thickness of $1 \mathrm{~mm}$, and the insulator was provided with a small groove to ensure normal processing of workpieces 1 and 2 . At the same time, workpiece 1 was blocked by a water shield to prevent the working fluid on the surface of the workpiece 1 from converging to the wire electrode from the gap between the insulator and the workpiece during processing, thus ensuring measurement accuracy. This experimental device is shown in Fig.4(a). At the beginning of the machining process, switches $\mathrm{S} 1$ and $\mathrm{S} 2$ are simultaneously closed, that is, the positive pole of the pulse power supply is connected to workpiece 1 and workpiece 2 at the same time. After the wire electrode is completely cut into the workpiece, the oscilloscope is used to collect the discharge waveform of workpiece 2 when the wire electrode is running downward. The test principle and measurement diagram are shown in Fig. 4 (b). 


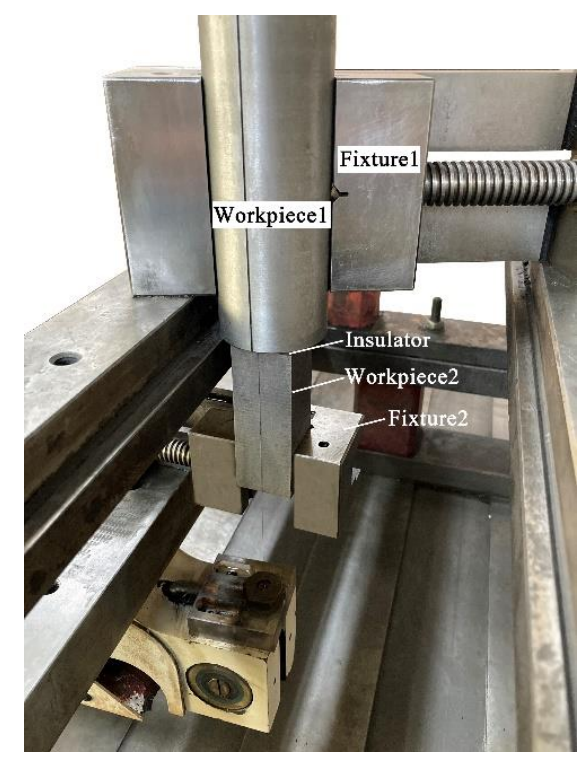

(a) Photograph of the experiment device

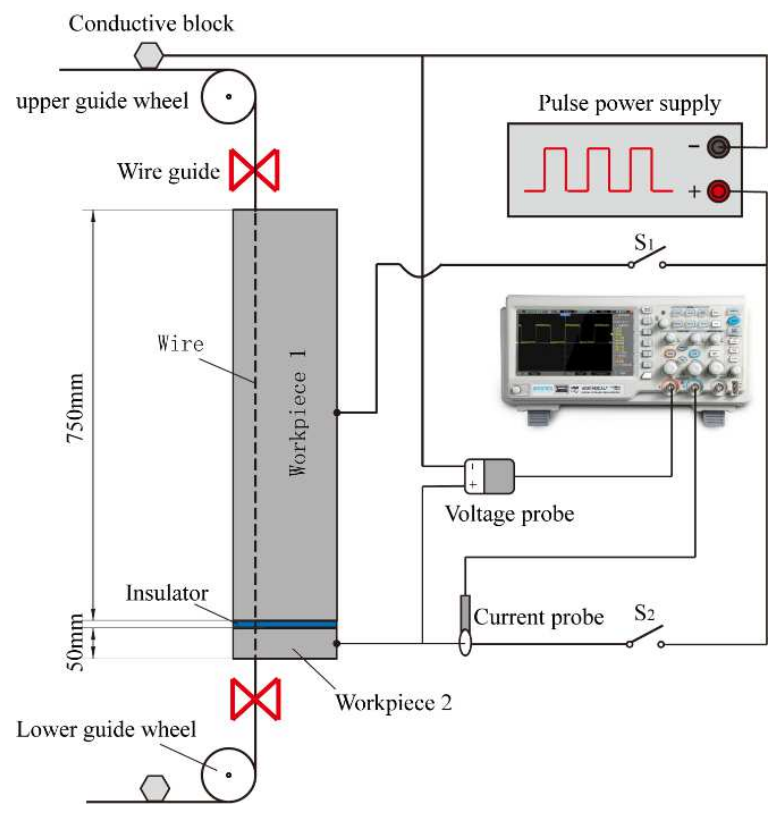

(b) Test principle and measurement diagram

Fig.4 Measurement scheme of the discharge waveform of workpiece 2

Figure 5 shows the discharge waveform of workpiece 2 during normal processing. Most of the waveforms are discharge waveforms without breakdown delay, and even appear in a short circuit state at the beginning of discharge, which indicates that the inter-electrode discharge environment is extremely poor [7].

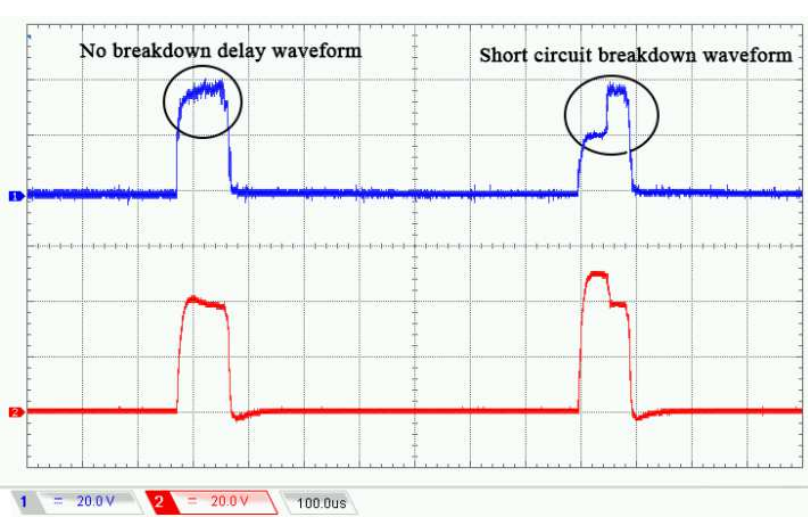

Fig.5 Discharge waveform of workpiece 2

According to the flow model of the inter-electrode working fluid, the flow of the inter-electrode working fluid can be increased by increasing the wire travelling speed and slit width. In the actual processing, because the wire travelling speed has reached $12 \mathrm{~m} / \mathrm{s}$, if we continue to improve the wire travelling speed, the cutting stability will be difficult to be guaranteed due to the aggravation of the mechanical vibration of the wire feeding system. Also, increasing the slit width can be achieved by increasing the discharge energy and conductivity of the working fluid. Increasing the discharge energy is to obtain a larger discharge gap [3], which is generally realized by using long pulse width and peak current. However, long pulse width and peak current will increase the discharge energy of a single pulse, and larger discharge energy will increase the vaporization of the working fluid; at the same time, the larger volume of erosion debris generated by large discharge energy is more difficult to be removed from the inter- electrode. Therefore, increasing the flow of the inter-electrode working fluid by increasing the discharge energy will make the inter-electrode environment worse, and increasing the conductivity of the working fluid may be a feasible method to increasing the flow of the inter-electrode working fluid.

\subsection{Effect of wire vibration}

For super-high thickness WEDM, the vibration of the wire electrode mainly includes low-frequency vibration caused by high-speed movement of the wire feeding system and high-frequency vibration caused by discharge explosion force. The first is the low-frequency vibration introduced by the wire feeding system with high-speed and reciprocating wire feeding. The 
vibration sources mainly include the reversal of the wire cylinder and the axial and radial runout of the guide wheel. Specifically, the wire vibration caused by the sudden change of the wire tension in the acceleration and deceleration stage of the motor during the wire cylinder reversing is mixed with the wire vibration caused by high-frequency discharge, which greatly reduces the machining stability. In addition, due to the lower feed speed of the wire electrode during the superhigh thickness machining process, the frequent reversal of the wire cylinder will inevitably lead to difficulties of maintaining the positional accuracy of the wire electrode, resulting in an uneven cutting surface. Secondly, to ensure that a single pulse has strong eroding ability, a larger discharge energy is generally used for machining, so the thin and flexible wire electrode is subjected to greater discharge explosion force in the narrow and long machining area. However, the anti-interference capability of the wire electrode is very low, and there is not enough working fluid between the electrodes to dampen the vibration of the wire electrode. Therefore, the positional accuracy of the wire electrode will significantly change in a short time due to the random discharge explosive force. When the positional accuracy of the wire electrode is low, the feed path of the wire electrode is not strictly in accordance with the theoretical straight path but swings forward in a predetermined straight direction, as shown in Fig.6. At this time, the machining surface will produce uneven steaks. In the case of severe vibration, the streaks even run through the entire cutting surface.

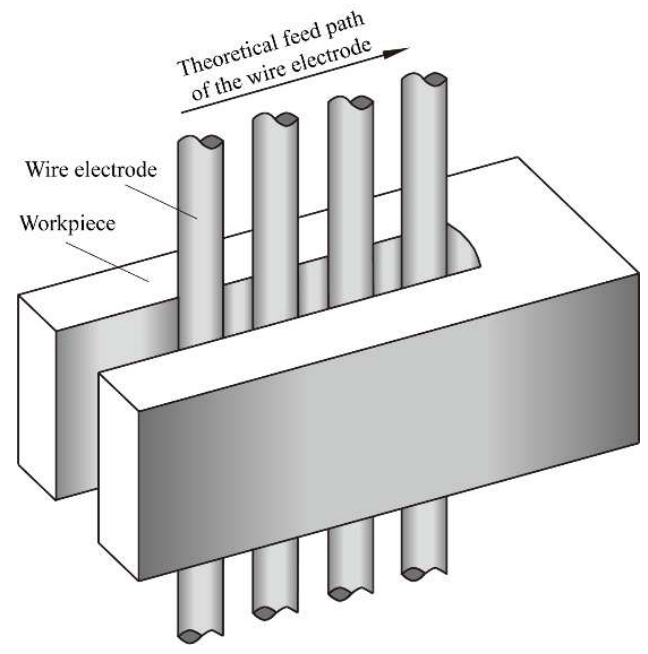

(a) Theoretical feed path

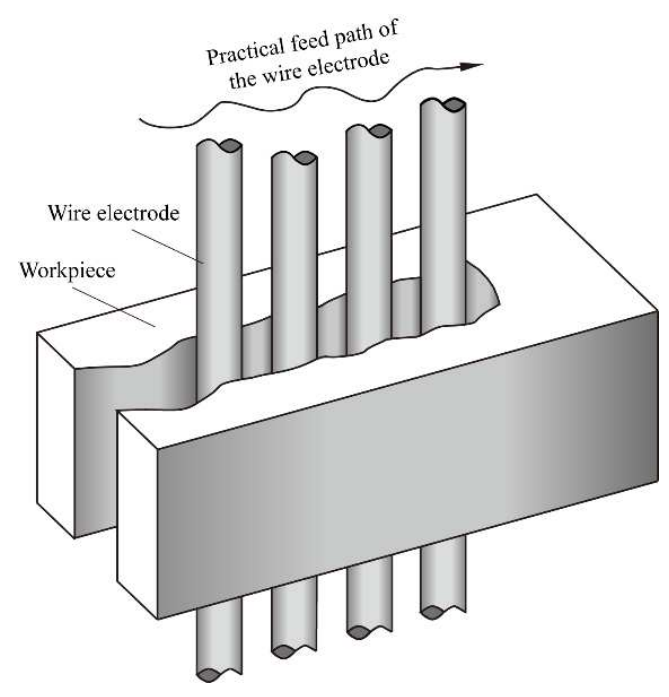

(b) Practical feed path

Fig.6 Feed model of the wire electrode ${ }^{[5]}$

\section{Measures to solve surface unevenness}

To obtain a flat cutting surface in super-high thickness machining, reduce the low-frequency and large amplitude vibration caused by high-speed wire feeding system and ensure that the working fluid is evenly filled in the inter-electrode, so that the wire electrode can be limited and dampened by the working fluid in the whole gap. Therefore, the positional accuracy of the wire electrode is relatively high during processing, and the wire electrode maintains stable feed at steady state.

\subsection{Wire guide and bidirectional tension device}

Wire guides and constant tension device are important means to improve the stability of wire electrode in processing area. In the past, the wire guide was not used in super-high thickness cutting. The reason is that it is generally believed that the wire guide is difficult to restrain the large and complex positional changes of the wire electrode, and the use of the wire guide will increase the friction of the wire electrode with high-speed travelling, resulting in decreased service life. Therefore, the vibration of the wire electrode cannot be effectively suppressed by low-frequency excitation sources such as the wire cylinder and the guide wheel. Therefore, the vibration of the wire electrode caused by the low-frequency excitation source such as the wire cylinder and the wire wheel cannot be effectively suppressed. The wire guide is primarily used to cut off 
the transmission path of wire vibration caused by the low-frequency excitation source to the processing area, so as to ensure the wire electrode can maintain high positional accuracy in the processing area. HS-WEDM has always existed for uneven wire tension ${ }^{[8]}$, while uneven wire tension will be more serious in super-high thickness processing. The gravity take-up with unidirectional tension can only alleviate uneven wire tension to a lesser extent due to the limited travel. Therefore, the gravity take-up with bidirectional tension is used to reduce the tension change of the wire electrode, and further reduce the positional change of the wire electrode during processing. Figure 7 shows the workpiece with thickness of $1000 \mathrm{~mm}$ machined by wire guide and the gravity take-up with bidirectional tension (other processing parameters are shown in Table 1). It can be seen from the figure that the deep streaks on the workpiece surface are obviously reduced, but there are still some shallow streaks, so more effective ways must be taken to improve the positional accuracy of the wire electrode.

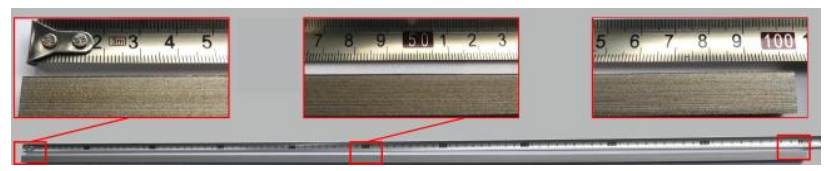

Fig.7 Workpiece with a thickness of $1000 \mathrm{~mm}$ machined by the wire guide and bidirectional tension device

\subsection{Improvement of the working fluid}

For super-high thickness workpiece machining, the key condition for continuous and stable machining is that the working fluid has evenly filled the interelectrode, which is also a key to improving the surface evenness ${ }^{[9]}$. The uniform working fluid can limit the interference of discharge explosive force on the wire electrode in the whole gap, so as to further improve the positional accuracy of the wire electrode during machining. To ensure that the working fluid can evenly fill the inter-electrode: first, the working fluid must have good cooling, washing, and deionization performance, which can then be brought into the slit as much as possible; second, it is necessary to reduce the vaporization of the inter-electrode working fluid as much as possible to ensure that there is enough working fluid to drive the debris out of the gap.

To verify that the improved working fluid JR3A (upgrade) can evenly fill the inter-electrode, a method of measuring leakage current was used to qualitatively evaluate the flow of the working fluid at the lower end of the slit. The experimental device for measuring the inter-electrode leakage current is shown in Fig.4(a). Since the probability of pulse discharge is very high when the super-high thickness cutting is stable, it is difficult to collect the no-load waveform, so the following test scheme is used to measure the leakage current waveform. At the beginning of processing, workpieces 1 and 2 are connected to the positive pole of pulse power supply at the same time. When the wire electrode is completely cut into the workpiece, the processing is suspended. Then, switch $\mathrm{S} 1$ is opened and switch S2 is closed, and only workpiece 2 is connected to the positive pole of the pulse power supply. Next, Resume processing that had been suspended but close the worktable feed. At this time, workpiece 1 is not processed, but only used as the channel for wire electrode travelling. The instantaneous leakage current waveform of workpiece 2 is collected. The measurement scheme is shown in Fig. 4 (b).

The higher the conductivity of the working fluid, the larger the discharge gap, and the greater the flow of the working fluid between the electrodes. Therefore, the improved working fluid JR3A (upgrade) has higher conductivity. In this experiment, the original working fluid JR1A and the improved working fluid JR3A (upgraded) were prepared to obtain two different working fluids. The conductivity of the two different working fluids were measured as shown in Table 2. The conductivity of the improved working fluid JR3A(upgrade) was 2.11 times that of the original working fluid JR1A. According to the above experimental scheme, two different working fluids were used to process under the same machining parameters. After processing, workpiece 2 was removed and cleaned, and its slit width was measured as shown in Fig.8. 
Table 2 Working fluid JR1A and JR3A (upgrade) related parameters

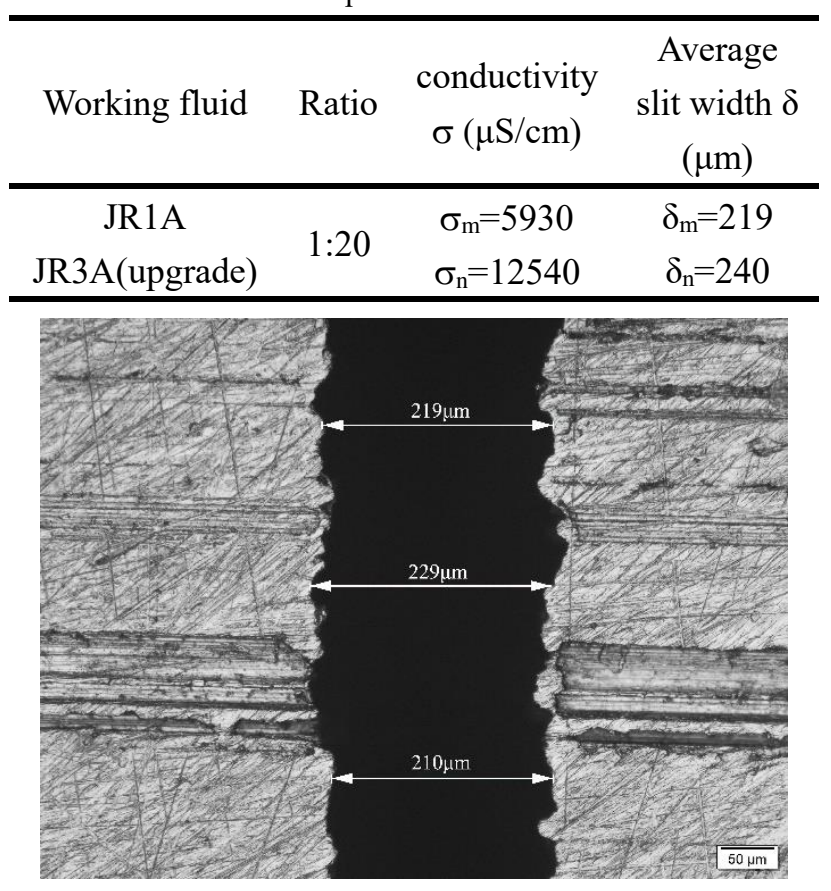

(a) Working fluid JR1A

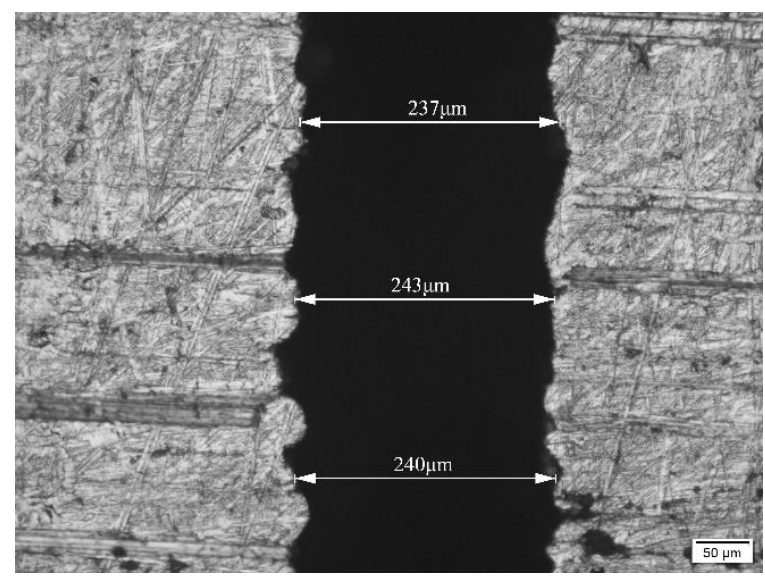

(b) Working fluid JR3A(upgrade)

Fig. 8 Slit machined with the different working fluid

Since the flow of the inter-electrode working fluid cannot be obtained by direct measurement, the flow is qualitatively analyzed by theoretical calculation and experimental verification. HS-WEDM was conducted using the working fluid with certain insulative performance. After the voltage was applied between the poles, the leakage current will be generated due to the conductivity of the medium. Under certain conditions, the inter-electrode leakage current can reflect the flow of inter-electrode working fluid ${ }^{[10]}$, and the leakage current is directly related to the inter-electrode working fluid resistance. Therefore, the inter-electrode working fluid resistance and leakage current can be used to explain the flow of inter-electrode working fluid in different working fluids for theoretical and practical comparison. The calculation formula of the interelectrode working fluid resistance $\mathrm{R}$ is expressed as ${ }^{[5]}$ :

$$
R=\frac{1}{\pi h \sigma} \ln \frac{r_{2}}{r_{1}}
$$

where $\mathrm{h}$ is workpiece thickness; $\sigma$ is the conductivity of the working fluid; $\delta$ is the slit width, $\delta=$ $2 \mathrm{r}_{2} ; \mathrm{r}_{1}$ is the wire radius.

Assuming that the inter-electrode working fluid resistance of JR1A and JR3A (upgrade) are $R_{m}$ and $R_{n}$, respectively, the data measured in Table 2 were substituted into formula (3), and the following results can be obtained:

$$
\begin{aligned}
& R_{m}=\frac{1}{\pi h \sigma_{m}} \ln \frac{\delta_{m}}{2 r_{1}}=5.2662 \Omega \\
& R_{n}=\frac{1}{\pi h \sigma_{\mathrm{n}}} \ln \frac{\delta_{n}}{2 r_{1}}=3.9149 \Omega
\end{aligned}
$$

The theoretical leakage current ratio of two different working fluids is as follows:

$$
\eta_{\mathrm{th}}=\frac{\mathrm{I}_{\mathrm{m}}}{\mathrm{I}_{\mathrm{n}}} \times 100 \%=\frac{R_{n}}{R_{m}} \times 100 \%=\frac{3.9149}{5.2662} \times 100 \%=74.34 \%
$$

In the experiment, the same machining parameters are used for cutting, and the oscilloscope is used to collect the voltage and current waveforms of workpiece 2 when processing in two different working fluids. The waveforms are shown in Figure 9. The leakage current can be read from the waveform, so the ratio of leakage current generated by two different working fluids can be obtained as follows:

$$
\eta_{\mathrm{ac}}=\frac{\mathrm{I}_{\mathrm{m}}}{\mathrm{I}_{\mathrm{n}}} \times 100 \%=\frac{1.0}{1.4} \times 100 \%=71.43 \%
$$

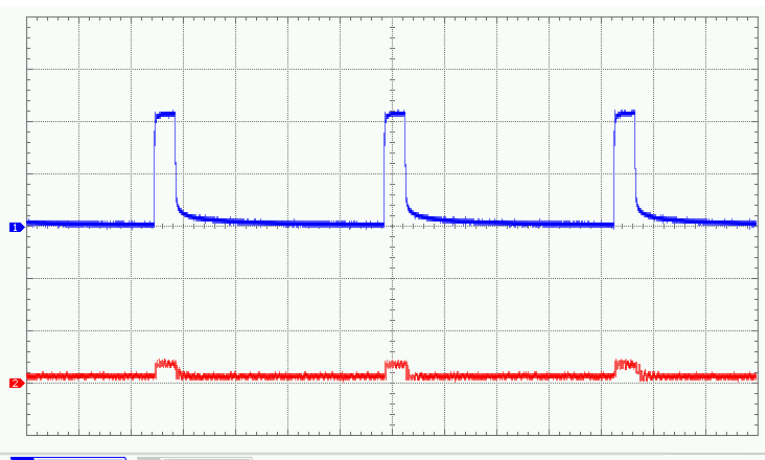

$=50.0 \mathrm{~V} / 2=2.0 \mathrm{~V}$

(a) Working fluid JR1A 


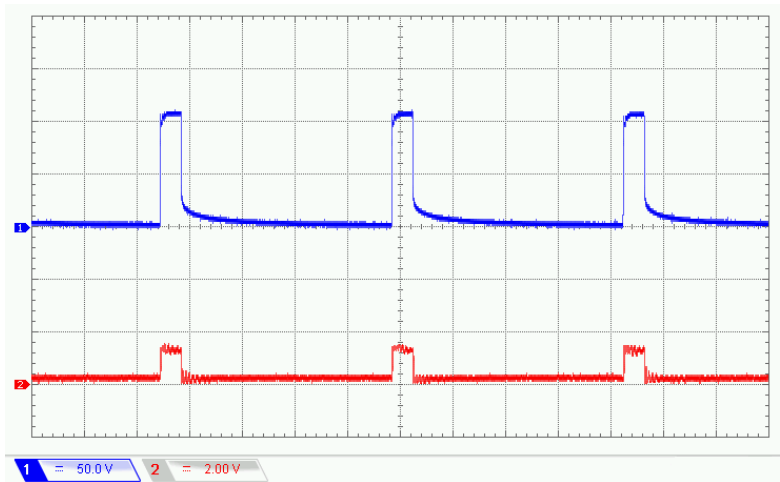

(a) Working fluid JR3A(upgrade)

Fig.9 Leakage current waveform machined with different working fluids

It can be seen from equation (2) that when the wire speed $v_{0}$ is constant, the flow $Q$ of the inter-electrode working fluid is related to the slit width $\delta$. It can be seen from equation (3) that when the workpiece thickness is fixed, the resistance of the inter-electrode working fluid is related to the conductivity $\sigma$ of the working fluid and the slit width $\delta$. Therefore, when the workpiece thickness and processing parameters are the same, there is a certain relationship between the resistance $R$ of the inter-electrode working fluid and the conductivity $\sigma$ of the working fluid and the working fluid flow Q. As the inter-electrode leakage current is related to the conductivity of the working fluid and the working fluid flow, and the conductivity of the two different working fluids are different, and it is difficult to determine whether the inter-electrode leakage current difference of the two working fluids is related to the working fluid flow. Therefore, assuming that the flow of the two working fluids is the same, the ratio of the leakage current generated by the conductivity difference between the two working fluid is as follows:

$$
\eta_{\text {th }}^{\prime}=\frac{R_{n}}{R_{m}} \times 100 \%=\frac{\sigma_{m}}{\sigma_{n}} \times 100 \%=\frac{5930}{12540} \times 100 \%=47.29 \%
$$

Owing to $\eta_{\text {th }}^{\prime}<\eta_{\mathrm{ac}}$, and $\eta_{\mathrm{th}}$ is close to $\eta_{\mathrm{ac}}$, the difference in leakage current between the two working fluids is determined by the conductivity $\sigma$ and flow $Q$ of the working fluid. The improved working fluid JR3A (upgrade) produces wider slits during machining. According to the flow model of the inter-electrode working fluid, the flow of working fluid JR3A (upgrade) is greater than that of working fluid JR1A during machining. Assuming that the flow of working fluids JR1A and JR3A (upgrade) are Q1 and Q2, respectively, the increase ratio of the outlet flow of the improved working fluid JR3A (upgrade) at the lower end of the workpiece to that of the working fluid JR1A is as follows:

$$
\omega=\frac{\mathrm{Q}_{2}-\mathrm{Q}_{1}}{\mathrm{Q}_{1}} \times 100 \%=\frac{\frac{\mathrm{r}_{1}^{2}-\left(\delta_{n} / 2\right)^{2}}{2 \operatorname{In}\left[\left(2 \mathrm{r}_{1}\right) / \delta_{n}\right]}-\frac{\mathrm{r}_{1}^{2}-\left(\delta_{m} / 2\right)^{2}}{2 \operatorname{In}\left[\left(2 \mathrm{r}_{1}\right) / \delta_{m}\right]}}{\frac{\mathrm{r}_{1}^{2}-\left(\delta_{m} / 2\right)^{2}}{2 \operatorname{In}\left[\left(2 \mathrm{r}_{1}\right) / \delta_{m}\right]}-\mathrm{r}_{1}^{2}} \times 100 \%
$$$$
=56.72 \%
$$

The outlet flow of the improved working fluid JR3A (upgrade) at the lower end of the workpiece is $56.72 \%$ higher than that of the working fluid JR1A. Therefore, the improved working fluid can more easily fill the inter-electrode.

Figure 10 shows a model of inter-electrode state corresponding to different working fluids in super-high thickness machining. It can be seen from Fig.10 (a) that the slit width $\delta_{\mathrm{m}}$ generated by discharge in working fluid JR1A is narrow and the flow of working fluid is less in the slit and continuously vaporized in the narrow and long discharge gap during machining. Therefore, less working fluid in the slit cannot dampen and absorb the vibration of the wire electrode. In addition, the ability of the working fluid to drive the debris out of the gap is greatly reduced (as shown by the arrow on the left side of the figure), resulting in unstable machining. The working fluid JR3A (upgrade) with higher conductivity, melting point and vaporization point components is just the opposite, and its model of the inter-electrode state is shown in Fig.10(b). Since the discharge can form a larger discharge gap, the slit width $\delta_{\mathrm{n}}$ will be wider, and the flow of working fluid in the slit will be more. Therefore, the dielectric fluid can play a very good damping effect on the wire electrode in the narrow and long gap. In addition, due to the increase of the components with a higher melting point and vaporization point in the working fluid, the vaporization of the inter-electrode working fluid is greatly reduced, which can ensure that there is sufficient working fluid in the gap. Therefore, its ability to carry the erosion products out of the gap is greatly improved (as shown by the arrow on the left side of the figure) ${ }^{[11]}$, which 
greatly improves the cutting stability. When the conductivity of the working fluid increases, the leakage current will increase accordingly, and more discharge energy will be consumed during processing; at the same time, the discharge gap will increase. Therefore, the cutting speed will be reduced, but the machining stability is the main goal, and the machining stability is the key factor to ensuring the surface evenness.

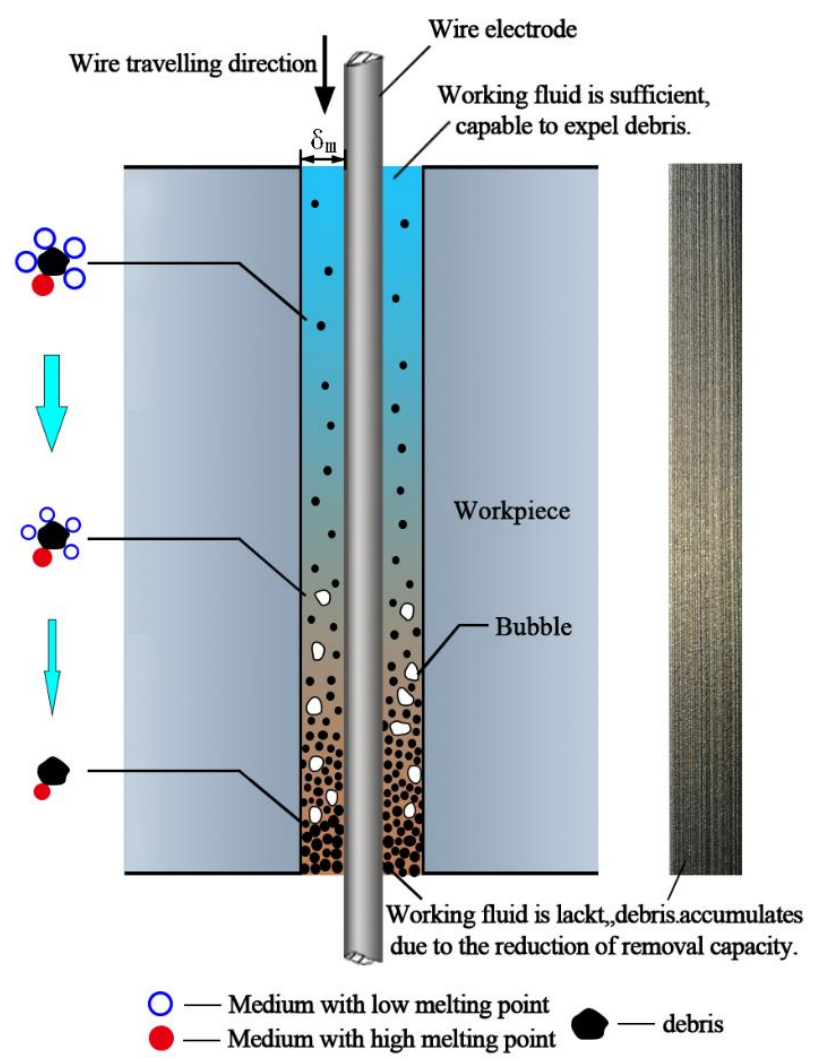

(a) Inter-electrode state of working fluid JR1A and debris expelling condition

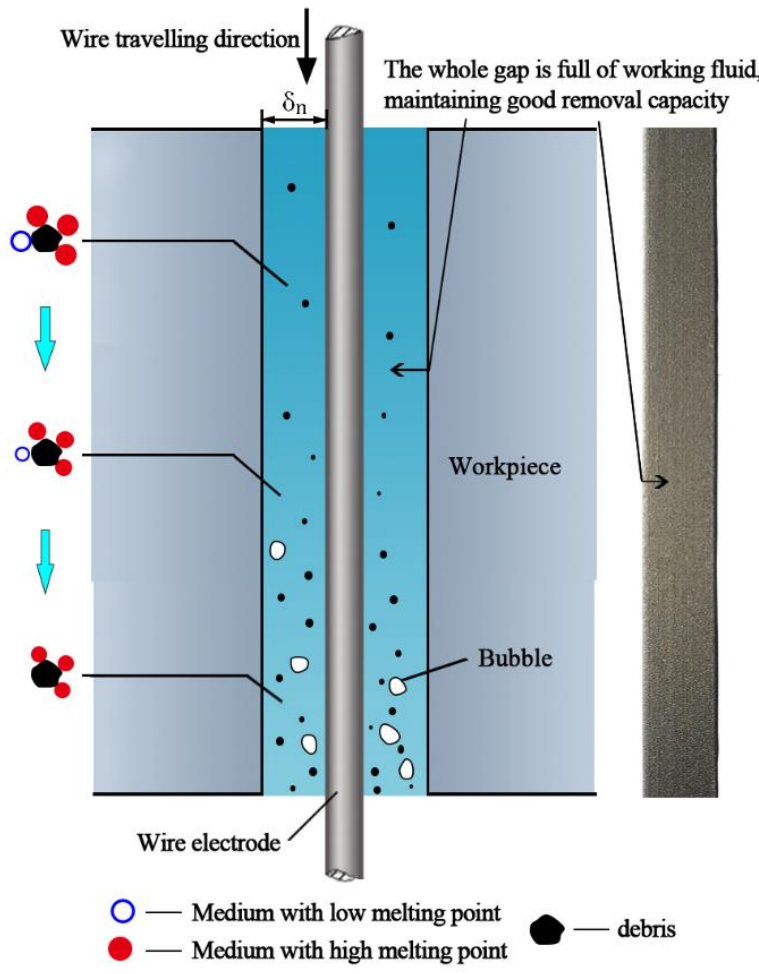

(b) Inter-electrode state of working fluid JR3A (upgrade) and debris expelling condition

Fig.10 Schematics of inter-electrode state in super-high thickness machining

\section{Sample of super-high thickness workpiece}

The improved system was used to cut a $1000 \mathrm{~mm}$ thick workpiece. Other parameters are shown in Table 1. Figure 11 shows the overall and partially magnified photograph of the workpiece. It can be seen that the surface evenness has been greatly improved. Under the same conditions, the pulse width was adjusted to $120 \mu \mathrm{s}$, the duty cycle was $1: 10$, the average cutting current was $3.5 \mathrm{~A}$, and the cutting test of the workpiece with a thickness of $2000 \mathrm{~mm}$ was carried out. The cutting efficiency was $3000 \mathrm{~mm}^{2} / \mathrm{h}$, and the surface roughness was $\mathrm{R}_{\mathrm{a}} 6.864 \mu \mathrm{m}$. Figure 12 shows a sample with a thickness of $2000 \mathrm{~mm}$.

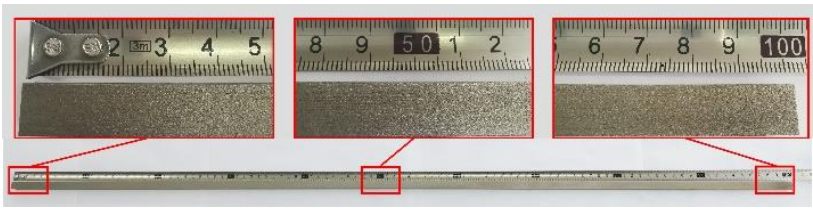

Fig.11 The overall and partially magnified photograph of a 1000 $\mathrm{mm}$ thick workpiece machined by the improved system 


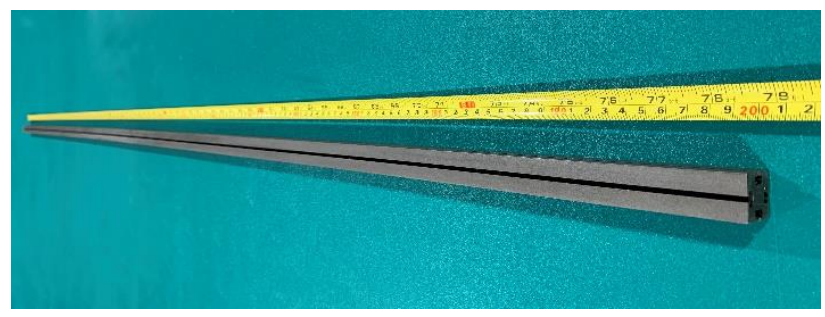

Fig.12 2000 mm workpiece

\section{Conclusions}

(1) For super-high-thickness workpiece machining, with increasing wire frame span, the rigidity of the wire electrode decreases. Under the action of discharge explosive force, the wire vibration greatly increases, and the cutting stability inevitably decreases, which will have a serious impact on the cutting surface evenness.

(2) The low-frequency vibration of the wire electrode can be reduced by using the wire guide and gravity take-up with bidirectional tension, thereby improving the cutting surface evenness.

(3) The improved working fluid with higher conductivity, melting point and high vaporization point can increase the flow of the working fluid in the slit. The sufficient fluid medium is used to dampen and absorb the vibration of the wire electrode in the gap, which plays a key role in improving the stability of super-highthickness workpiece machining.

\section{Declarations}

Acknowledgments The authors extend their sincere thanks to those who contributed in the preparation of the instructions.

Funding Information This work was supported by the National Natural Science Foundation of China (Grant No.51975290).

Conflicts of interest The authors have no relevant financial or non-financial interests to disclose.

Ethical approval Not applicable.

Consent to participate Not applicable.

Consent to publish Not applicable.

Authors Contributions All authors have been personally and actively involved in substantive work leading to the report.

Code availability Not applicable.
Availability of data and materials The data and materials set supporting the results are included within the article.

\section{References}

[1] China Society of mechanical engineering nontraditional machining branch (2016). Nontraditional manufacturing technology roadmaps[M]. China Science and Technology Press, Beijing, pp 48-50.

[2] Liu ZD (2013) The present situation and development of high-speed reciprocating wire EDM. Mach Manuf Automa 42:1-6.

[3] Li MH, Bu FL (1996) Theoretical research on super-high thickness of WEDM [J]. Electromach Mould 30(9):122-126.

[4] Wan ZY (2008) Research on super-large thickness machining technology of HS-WEDM [D]. Guangdong University of Technology 2008:2-5.

[5] Li CR, Liu ZD, Fang LJ, Pan HW, Qiu MB (2017) Super-high-thickness high-speed wire electrical discharge machining. Int $\mathrm{J}$ Adv Manuf Technol 2017:1-14

[6] Liu ZD (2006) Research on cooling liquid used in super-high thickness workpiece cutting of HSWEDM. Electromach Mould 1:25-27.

[7] Zhang YQ, Liu ZD, Pan HW (2017) Dielectric fluid lifespan detection based on pulse discharge probability in wire electrical discharge machining[J]. Int J Adv Manuf Technol.

[8] Li LL, Liu ZD, Li XF, Li MM (2014) Non-even wire tension in high-speed wire electrical discharge machining. Int J Adv Manuf Technol 78(1-4):503-510.

[9] Liu ZD (2005) Research on the general feature of emulations \& dielectrics and its tendency in 
WEDM-HS. Aviat Precis Manuf Technol 41:38-42.

[10] Liu ZD, Wang ZX (2011) Research on high speed wire-cut electrical discharge machine based on analysis of inter-electrode flow. Journal of Shanghai Jiaotong university 45(001):61-65.

[11] Pan HW, Liu ZD, Li CR (2017) Enhanced debris expelling in highspeed wire electrical discharge machining. Int J Adv Manuf Technol 93:29132292. 
Figures

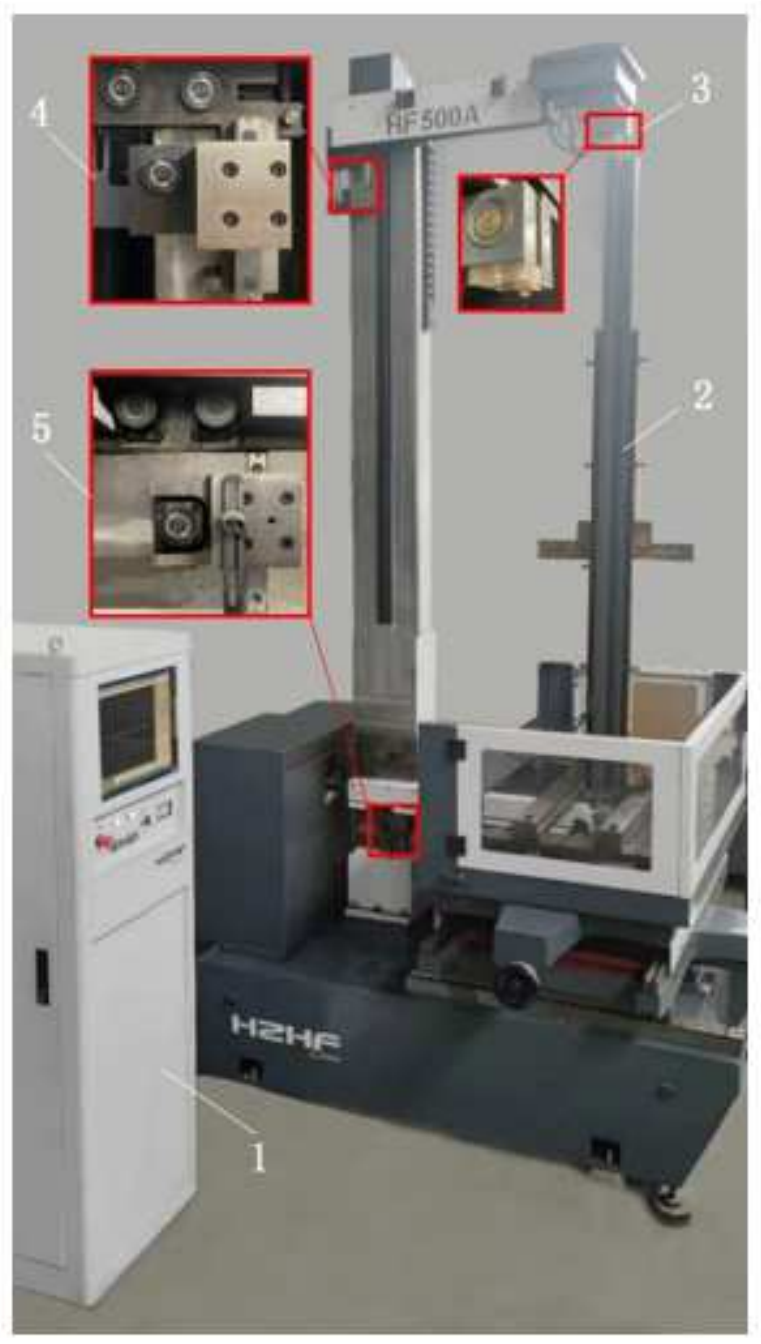

Figure 1

Photograph of the experimental equipment

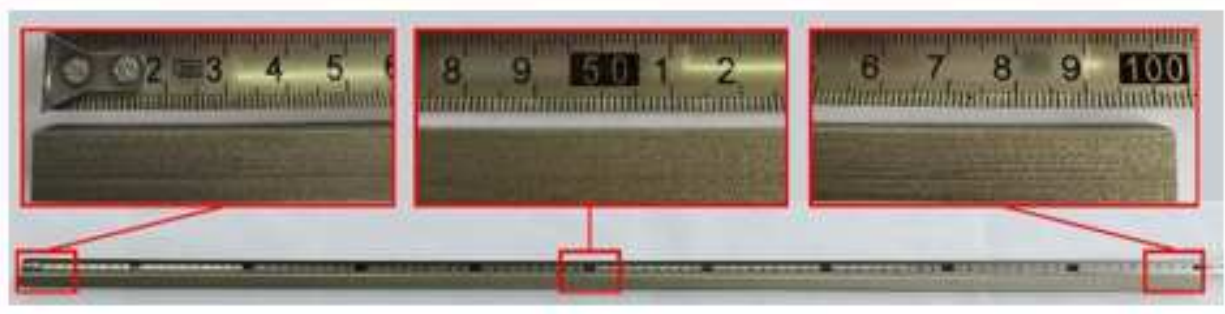

Figure 2

The overall and partially magnified photograph of the $1000 \mathrm{~mm}$ thick workpiece 


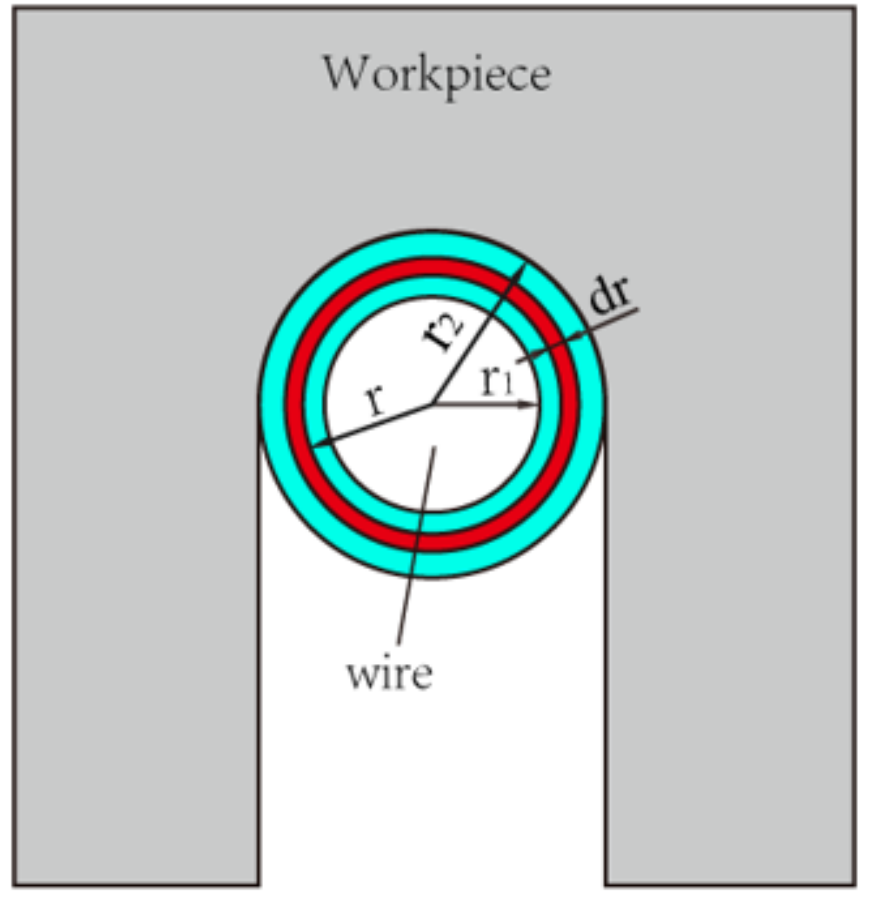

Figure 3

Flow model of the working fluid in the annular gap of a concentric cylinder

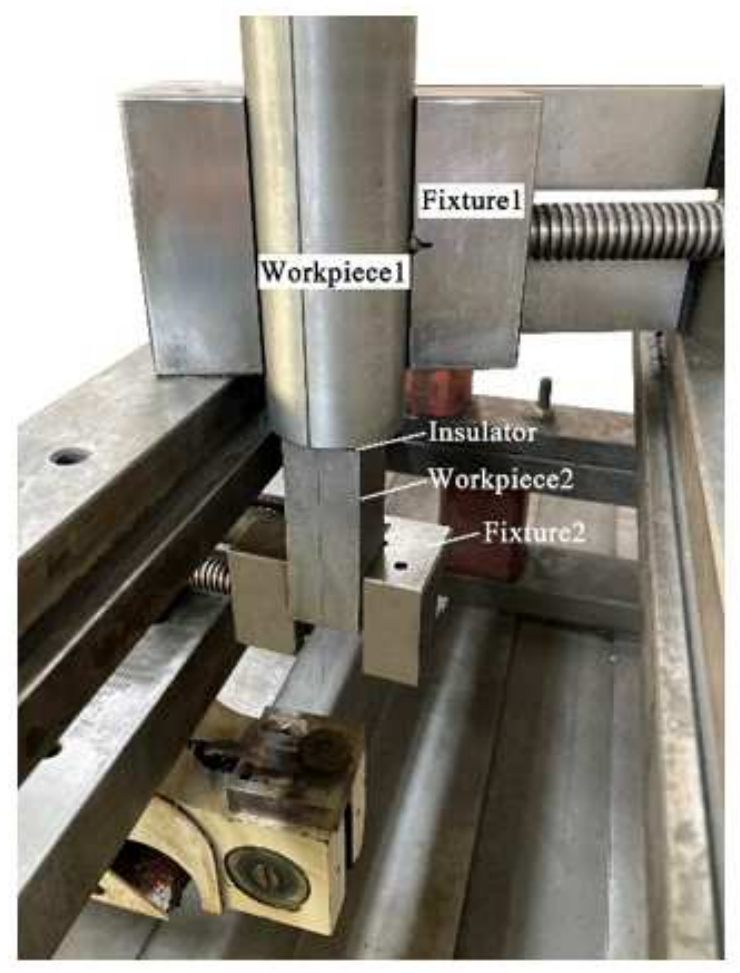

(a) Photograph of the experiment device

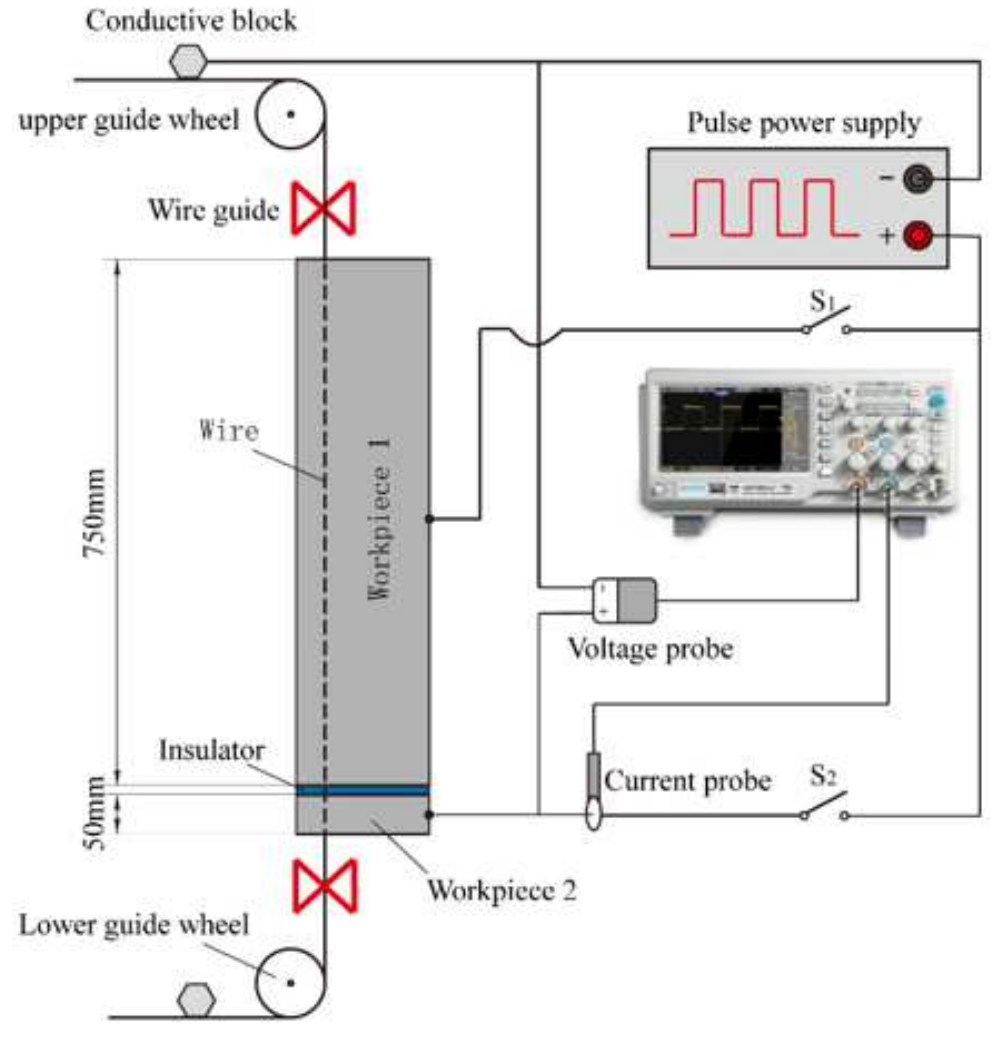

(b) Test principle and measurement diagram

Figure 4 


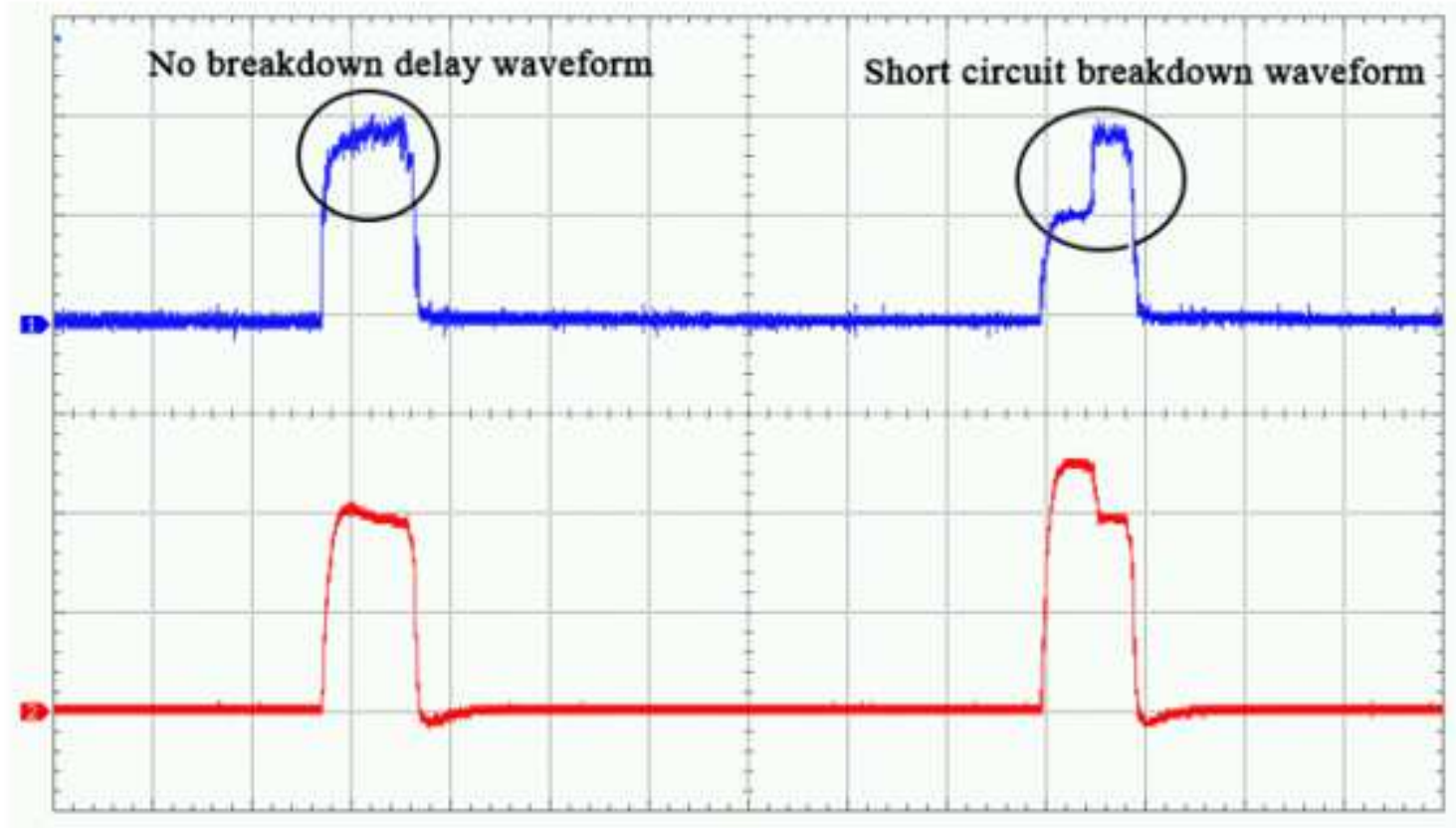

$1=20.0 \mathrm{~V}-2 \mathrm{~V}=200 \mathrm{~V} \quad 1000 \mathrm{us}$

\section{Figure 5}

Discharge waveform of workpiece 2

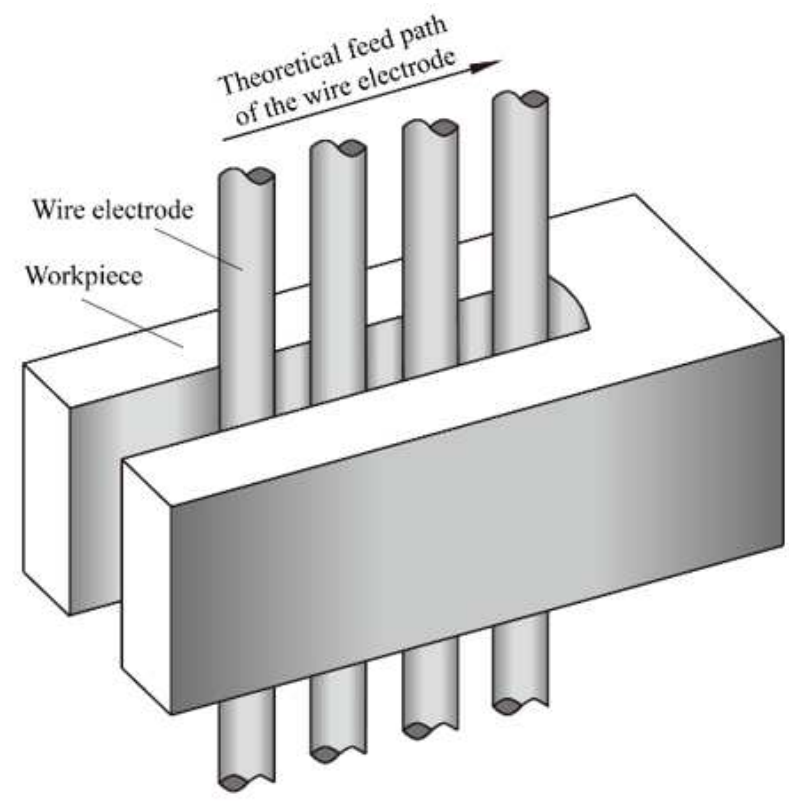

(a) Theoretical feed path

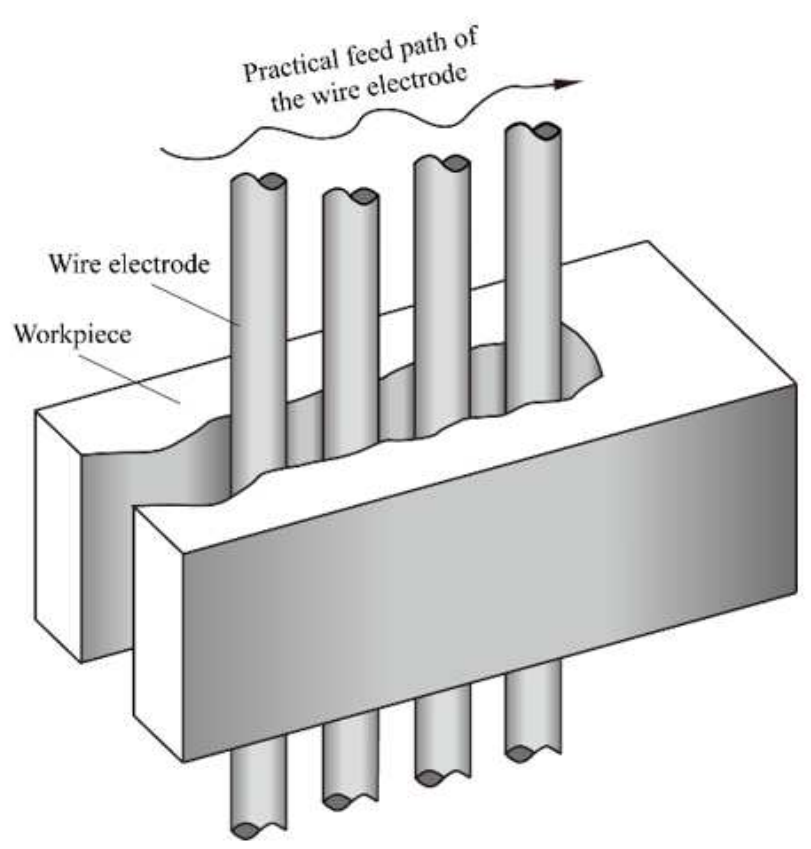

(b) Practical feed path

Figure 6

Feed model of the wire electrode [5] 


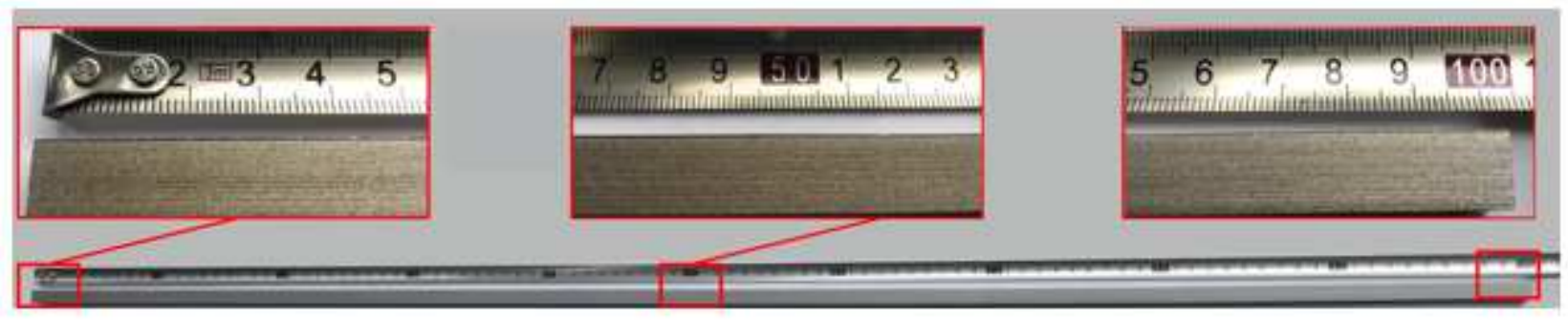

\section{Figure 7}

Workpiece with a thickness of $1000 \mathrm{~mm}$ machined by the wire guide and bidirectional tension device

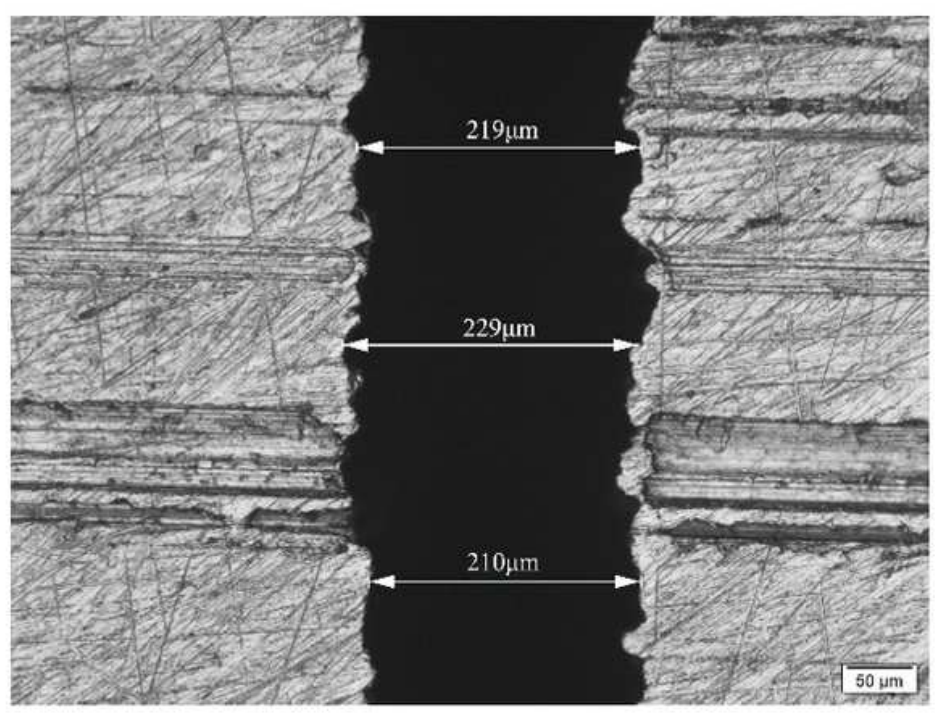

(a) Working fluid JR1A

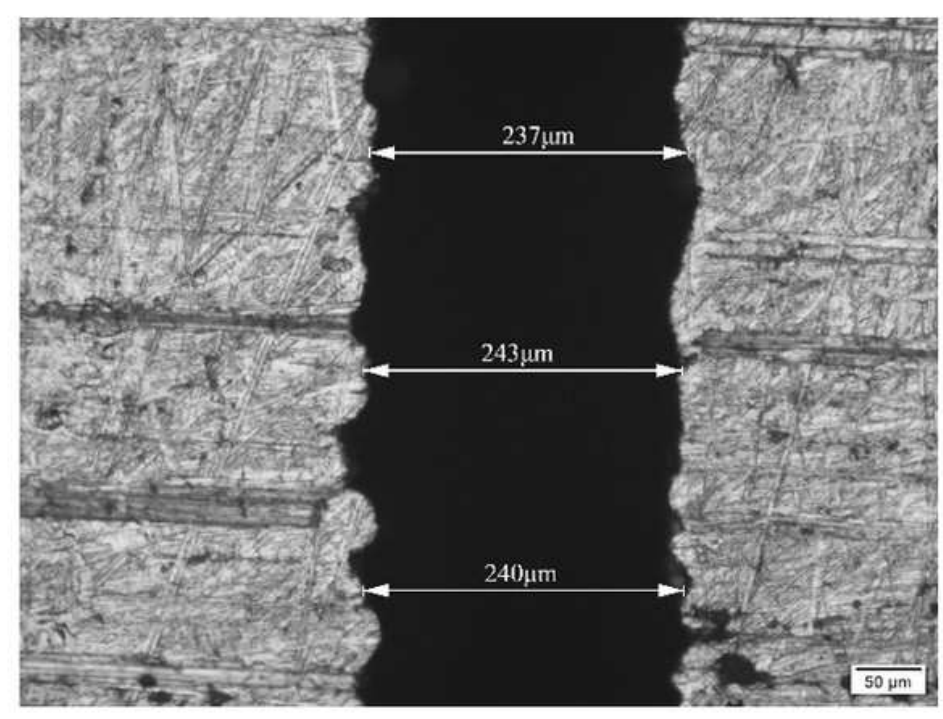

(b) Working fluid JR3A(upgrade)

\section{Figure 8}

Slit machined with the different working fluid

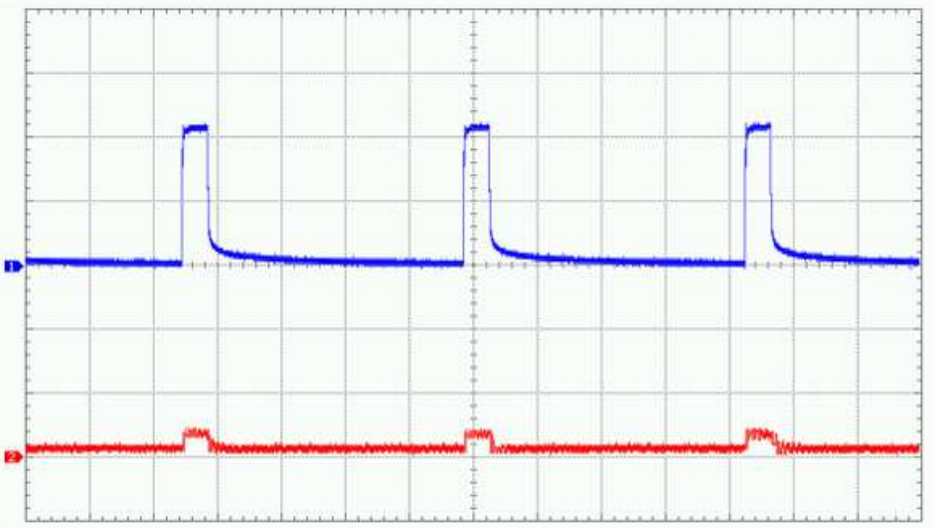

U-500v $2=200 \mathrm{~V}$

(a) Working fluid JR1A

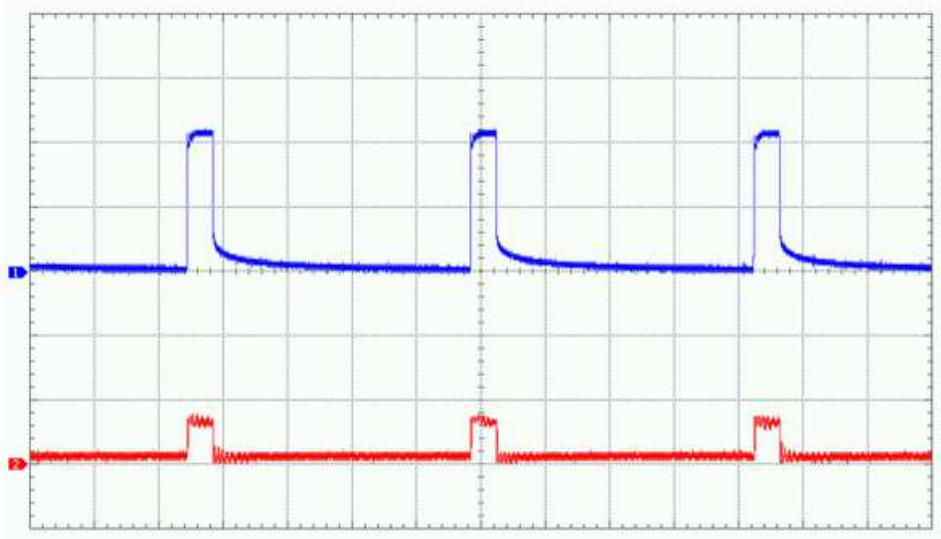

$\mathbf{1}=50.0 \mathrm{~V} 2=200 \mathrm{~V}$

(b) Working fluid JR3A(upgrade) 


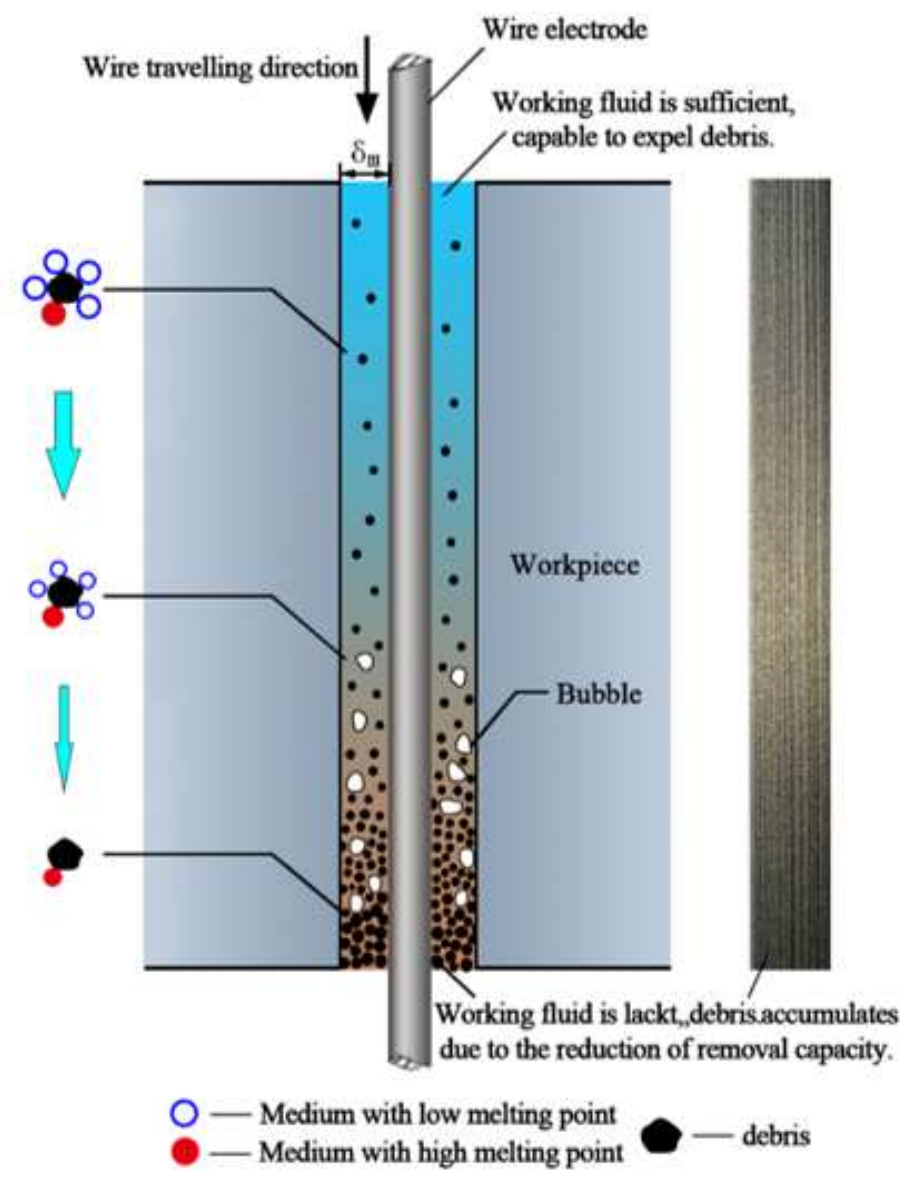

(a) Inter-electrode state of working fluid JR1A and debris expelling condition

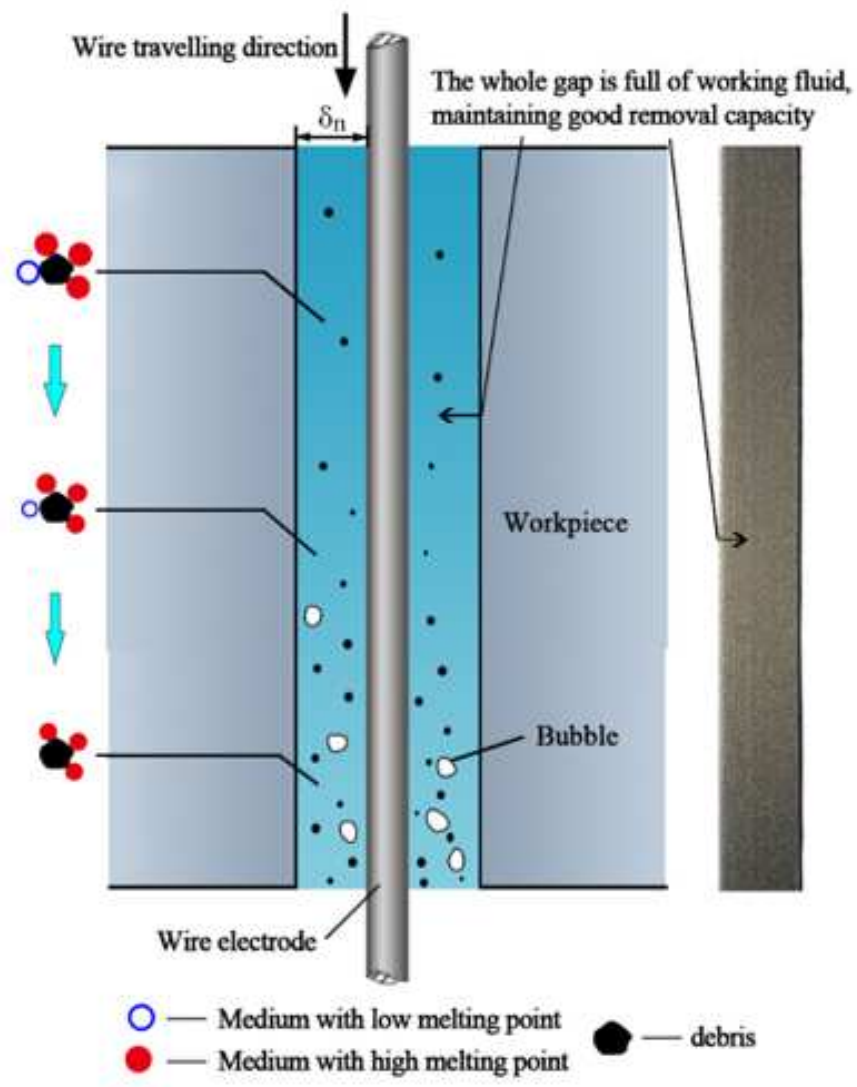

(b) Inter-electrode state of working fluid JR3A (upgrade) and debris expelling condition

\section{Figure 10}

Schematics of inter-electrode state in super-high thickness machining

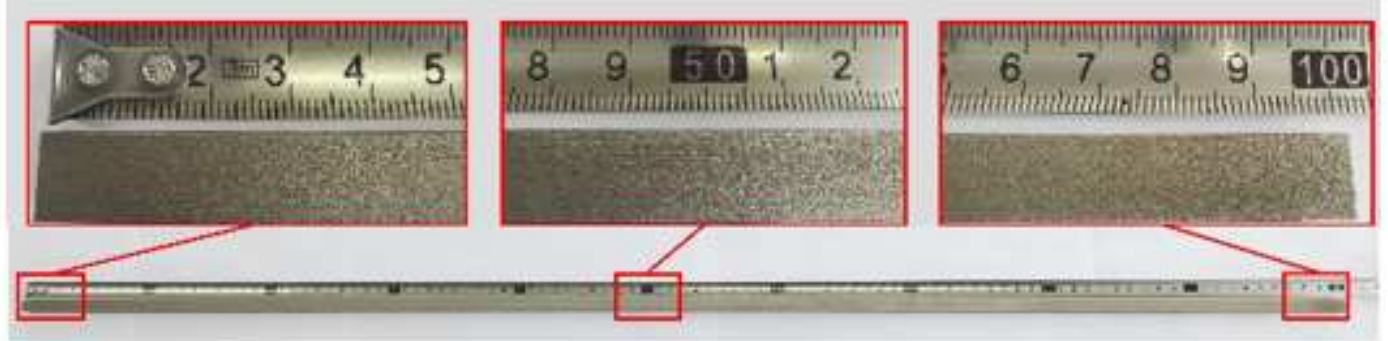

\section{Figure 11}

The overall and partially magnified photograph of a $1000 \mathrm{~mm}$ thick workpiece machined by the improved system 


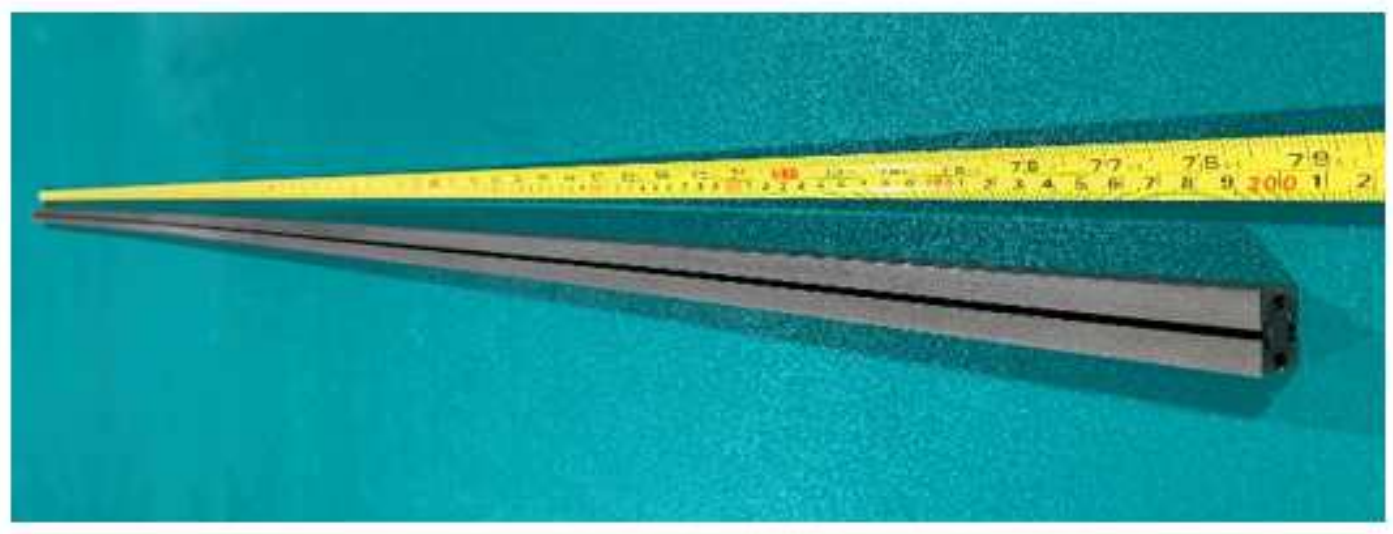

Figure 12

2000 mm workpiece 